\title{
Staphylococcus aureus sensíveis à meticilina provenientes de leite mastítico no Estado do Rio de Janeiro
}

\author{
Methicillin susceptible Staphylococcus aureus from mastitic milk \\ in Rio de Janeiro State, Brazil
}

\author{
Olney Vieira-da-Motta, ${ }^{*}$ Dirlei Molinari Donatele, ${ }^{*}$ Patrícia Ribeiro de Oliveira, ${ }^{* *}$ Marcio Manhães Folly*(1)
}

\begin{abstract}
Resumo
Em um total de 364 vacas portadoras de mastite subclínica e 1398 quartos mamários examinados, 555 amostras de leite foram coletadas durante os anos de 1996 e 1997 de 64 propriedades, em 15 municípios da região Norte/Noroeste do Estado do Rio de Janeiro. As amostras de leite foram testadas a partir de CMT (California Mastitis Test) e 128 cepas de Staphylococcus aureus foram isoladas e identificadas após análise de rotina laboratorial. Antibiogramas foram realizados, testando-se 15 antibióticos diferentes para definir o padrão de sensibilidade. Nos testes para os b-lactâmicos $96,1 \%$ das cepas foram resistentes (R), nenhuma cepa se enquadrou na faixa intermediária ( I ) e 3,9\% mostraram sensibilidade (S) à penicilina; seus derivados semi-sintéticos foram oxacilina $87,0 \mathrm{R}, 5,3 \%$ I e 7,6\% S; ampicilina 99,2 R e 0,8\% S e nenhuma intermediária, amoxicilina $88,2 \%$ R, $9,8 \%$ I e 2,0 S; para os b-lactâmicos cefalotina $96 \%$ S, 0,9\% R, 3,1\% I e cefoxitina $71,6 \%$ S, $5,5 \%$ R e $22,8 \%$ I. Todas as cepas foram $100 \%$ sensíveis à gentamicina, sulfazotrim e vancomicina. Nenhuma das cepas isoladas foi resistente à meticilina quando utilizada a concentração padrão de $25 \mathrm{mg} / \mathrm{mL}$ da droga para testes de cepas de origem humana (clone brasileiro MRSA III::B:A)
\end{abstract}

Palavras-chave: mastite bovina; meticilina; resistência; Staphylococcus aureus.

\begin{abstract}
From 364 subclinical mastitic dairy cows and 1398 quarters examined, a total of 555 milk samples were collected during 1996 and 1997 in 64 dairy farms in 15 municipalities of Northern State of Rio de Janeiro, Brazil. All samples were CMT (California Mastitis Test) tested and 128 strains of Staphylococcus aureus identified after routine laboratorial analyses. Fifteen different antibiotic tests were performed and susceptibility profiles of the strains were evaluated. For beta-lactamic, $96,1 \%$ of the strains were resistant $(R)$, $3,9 \%$ susceptible (S) and none was intermediary ( 1 ) to Penicillin. Towards penicillin semi-synthetic beta-lactamics, the strains showed $87 \%$ R, $7,6 \%$ S and $5,3 \%$ I to Oxacillin, $99,2 \% R, 0,8$ S $0 \%$ l to Ampicillin and $88,2 \%, 2,0 \%$ S and $9,8 \%$ l to Amoxicillin. Towards Cephalotin, $96 \%$ of strains were S, 3,1\%R, and 0,9\%l. For Cephoxitin the figures were $71 \%$ S, $5,5 \%$ R and $22,8 \%$ l. All strains tested $(100 \%)$ were susceptible to Gentamycin, Sulphazotrim and Vancomycin. None of the Staphylococcus aureus strains isolated were Methicillin-resistant to $25 \mathrm{mg} / \mathrm{mL}$ a dose used to test $S$. aureus strains from human origin (Brazilian MRSA III::B:A)
\end{abstract}

Keywords: bovine mastitis, methicillin, resistance, Staphylococcus aureus.

\section{Introdução}

Mastites são processos inflamatórios agudos ou crônicos das glândulas mamárias, caracterizados pelo aumento de células somáticas no leite, proveniente da glândula afetada e considerada a doença de maior custo aos criadores de gado de leite, resultando em perdas econômicas (DeGraves e Fetrow, 1993 ; Germano e Germano, 1995).

Os quadros de mastite prolongada ou crônica levam a contínua mudança na constituição dos tecidos da glândula e tecidos produtores de leite que são substituídos por tecidos conjuntivos ou fibrosos tornando o animal economicamente inviável. A enfermidade do úbere é a forma que os tecidos da glândula mamária respondem à injúria provocada.
Conseqüentemente, ocorre uma série de mudanças nos referidos tecidos, em conjunto com mudanças organolépticas no leite (gosto, odor, cor e consistência). O processo inflamatório da glândula mamária tem como função a eliminação da injúria e a devolução dos tecidos do úbere ao seu desempenho normal. Um dano leve da glândula mamária é rapidamente recuperável, caso não seja repetido. As injúrias mais graves têm por conseqüência a destruição do tecido produtor de leite no úbere (Saran, 1986).

A mastite subclínica, que mais nos interessa, é assim chamada por sua natureza inaparente, ou seja, geralmente não se distinguem sinais externos da enfermidade na vaca afetada. O animal aparentemente está normal em seu comportamento, o úbere está sem sinais de anormalidade e o mesmo

\footnotetext{
*Laboratório de Sanidade Animal-CCTA- Universidade Estadual do Norte Fluminense-UENF.

**Laboratório de Biologia Mol.de Bactérias-IMPPG-CCS-UFRJ.

(1)Autor para correspondência UENF - LSA - Centro de Ciências e Tecnologias Agropecuárias-Av. Alberto Lamego, 2000 - Horto - CEP $28015-620$ Campos dos Goytacazes -RJ.
} 
pode-se dizer do leite. Esta enfermidade é, geralmente, de longa duração e pode afetar a vaca durante toda sua vida produtiva, caso não sejam tomadas medidas para curá-la, podendo ocorrer uma perda de 10 a $26 \%$ dos quartos infectados (Saran, 1986).

Atualmente, cerca de 23 espécies de Staphylococcus são reconhecidas dentre elas Staphylococcus aureus, permanece como sendo um patógeno comum na glândula mamária (Langlois et al., 1990). Entretanto, as espécies coagulasenegativas também podem estar envolvidas em quadros de mastite (Hummel e Lehmann, 1994 ; Tyler, 1996). No Brasil foi detectado um índice de $38 \%$ de Staphylococcus coagulasenegativa em rebanhos no Estado do Paraná (Filippsen, et al., 1996). Trabalhos mais recentes mostram a importância da participação de $S$. aureus em quadros crônicos de infecções intramamárias e a dificuldade em combater a bactéria mesmo através de combinações entre drogas mais potentes in vitro, reafirmando os resultados de trabalhos prévios da natureza intratável dessas infecções causadas por S.aureus (Owens et al., 1997).

Os antibióticos beta-lactâmicos atuam através da inibição de enzimas envolvidas no processo de formação da parede da célula bacteriana. Estas enzimas são encontradas na membrana e catalisam a reação de ligação cruzada entre os polímeros de peptideoglicanos. Esta ligação cruzada promove uma rigidez adicional na parede da bactéria, que é essencial para a célula. Muitas dessas enzimas ligam-se covalentemente aos antibióticos beta-lactâmicos em seus sítios ativos e têm sido denominadas proteínas penicilina-ligadas ou PBP "Penicillin-binding protein" que são encontradas num total de cinco tipos de PBPs (1,2,3,3' e 4). A diferença fundamental entre Staphylococcus spp susceptíveis e meticilina-resistente está nas suas PBPs. A resistência à meticilina está associada com a produção de uma única PBP que não está presente em Staphylococcus spp susceptíveis. O determinante genético que confere a resistência a meticilina é denominado $m e c$, que é cromossoma-derivado. Foi mostrado que o gene para PBP2a faz parte do determinante mec que indica ser um gene altamente conservado (Hackbarth e Chambers, 1989).

Mais de $95 \%$ dos pacientes humanos com infecções estafilocócicas em todo o mundo não respondem ao tratamento com antibióticos de primeira linha tais como penicilina e ampicilina (Neu, 1992). A detecção de cepas de $S$. aureus meticilina-resistentes (MRSA) entre amostras isoladas de humanos é extremamente importante por se tratar de cepas potencialmente perigosas para pacientes internados em hospitais, podendo levar à morte em casos de infecção hospitalar (Teixeira et al., 1995) e mesmo para indivíduos recém-operados, carreadores de $S$. aureus na mucosa nasal correrem riscos de prolongamento no tempo de internação ao óbito devido a infecções por esta bactéria (Belkum et al., 1997). A participação de MRSA e $S$. aureus sensiveis a meticilina (MSSA) em infecções hospitalares na cidade de New York mereceu atenção para as possíveis perdas econômicas relacionadas àquelas cepas. Os autores constataram que, embora os custos médios diretos com pacientes infectados com MRSA e MSSA fossem similares, a ocorrência de óbitos devido a MRSA foi mais elevada (21\% versus $8 \%$ ) (Rubin et al., 1999).

Neste trabalho foi feita a identificação do $S$. aureuis utilizando técnicas bacteriológicas de rotina de isolamento visando auxílio em observações epidemiológicas. As cepas de $S$. aureus foram testadas segundo Owens e Watts (1987) para observação de seu perfil de sensibilidade a antimicrobianos, analisados e comparados com a cepa do clone brasileiro MRSA III::B:A através da técnica de triagem em placas com uma concentração de $25 \mathrm{mg} / \mathrm{ml}$ de meticilina padronizado para cepas de origem humana para fins epidemiológicos (De Lancastre et al., 1991).

\section{Material e métodos}

Em um total de 364 vacas portadoras de mastite subclínica e 1398 quartos mamários examinados, 555 amostras de leite foram coletadas durante os anos de 1996 e 1997 de 64 propriedades, em 15 municípios da região Norte/Noroeste do Estado do Rio de Janeiro e identificadas a partir do CMT (California Mastitis Test) com uso de reagente comercial (CMTFATEC). Deste total, foram isoladas 128 amostras de $S$. aureus. Em adição, uma amostra-padrão (ATCC-25923 American Type Culture Collection) foi utilizada como controle positivo e uma amostra como controle negativo (Staphylococcus schleifferi) classificada no LSA através do Kit ApiStaph (Biomérieux, cód.20500). As amostras de leite coletadas foram transportadas sob refrigeração até o laboratório, onde foram incubadas a $37^{\circ} \mathrm{C}$. Após 18 horas as amostras foram estriadas com auxílio de alça de platina em ágar seletivo de Chapman (Merck, cat. $\mathrm{n}^{\circ}$ 1.05469) e posteriormente as Unidades Formadoras de Colônias (UFCs) isoladas foram inoculadas em ágar sangue (Merck cat. $n^{\circ}$ 1.10886) suplementado com $5 \%$ de sangue desfibrinado de carneiro para observação do padrão hemolítico e em agar DNAse (Merck cat. no 1.10449) para identificação de cepas DNAse positivas (formação de halo ao redor das UFCs) após inundação da placa com ácido clorídrico $(\mathrm{HCl} 1,5 \mathrm{~N})$. Nestas etapas de cultivo em ágar, as amostras foram incubadas a $37^{\circ} \mathrm{C}$ por 24 horas para observação do crescimento de Staphylococcus sp. A seleção das UFCs de Staphylococcus aureus obedeceu aos critérios de morfologia, pigmentação, capacidade hemolítica e produção de DNAse. Das UFCs isoladas foram preparados esfregaços para serem corados pelo método de Gram para verificação de suas características morfotintoriais. As UFCs que se revelaram como cocos Gram-positivos em forma de cachos foram submetidas aos testes de fermentação do manitol (Merck, cat no 5404) em anaerobiose e de produção da catalase, oxidase (Difco, cat. $n$ ㅇ 3530-31) e coagulase (Difco, cat. $n \circ 3530-31$ ). Para este último, UFCs provenientes de placas foram inoculadas em $5,0 \mathrm{~mL}$ de caldo TSB e incubadas a $37^{\circ} \mathrm{C}$ sob agitação, de onde foram, então, retiradas duas gotas de inóculo e adicionadas em tubo contendo $0,5 \mathrm{~mL}$ de plasma de coelho tratado com citrato. Os tubos foram, então, incubados a $37^{\circ} \mathrm{C}$ em um intervalo de tempo que variou de 2-24 horas, a fim de observar a formação de coágulo; também foi utilizado o kit Bacto Staph Latex Test (Difco, cat. 3850-32-7) que analisa a produção de proteína A e coagulase por $S$. aureus. Em casos de resultados não conclusivos pelos métodos citados acima, o kit Api Staph (Biomérieux, cód. 20500) foi utilizado segundo os métodos descritos pelo fabricante.

\section{Análise do perfil antimicrobiano}

O método de Bauer et al. (1966) foi realizado após a identificação das cepas de S.aureus e incubadas a $37^{\circ} \mathrm{C}$ por 24 horas em caldo TSB (Tryptic Soya Broth) (Difco, cat. no 0370-01-1) e em seguida as amostras foram diluídas em TSB com concen- 
tração ajustada à escala 0,5 de McFarland, correspondente a $1,5 \times 10^{8}$ UFCs, e inoculadas com swab estéril em placas contendo ágar Mueller Hinton (Merck cat. № 5437). Foram utilizados multidiscos de antibióticos (Laborclin, reg. M.S. no100970.10020) para Gram positivos, contendo os seguintes antibióticos: penicilina G (PEN, 10U), oxacilina (OXA, 1 $\mu \mathrm{g}$ ),

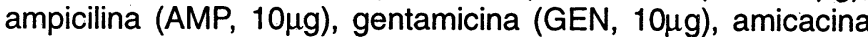

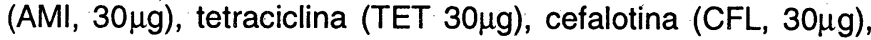
cefoxitina (CFO, $30 \mu \mathrm{g}$ ) eritromicina (ERI, $15 \mu \mathrm{g})$, clindamicina (CLI, $2 \mu \mathrm{g}$ ), lincomicina (LIN, $2 \mu \mathrm{g})$, sulfazotrim (SUL, $25 \mu \mathrm{g}$ ),

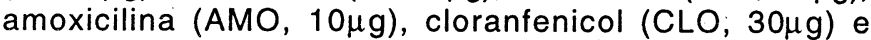

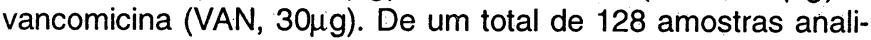
sadas, foram observadas diferentes respostas (Quadro 1).

\section{Teste de Resistência a Meticilina (Met-25)}

Primeiramente, as células foram cultivadas em $2,0 \mathrm{~mL}$ de TSB (Difco, cat. no 0370-01-1), no Laboratório de Sanidade Animal C.C.T.A/U.E.N.F., a $37^{\circ} \mathrm{C}$ por 24 horas, em banho-maria sob agitação. As amostras foram transportadas em gelo para o Laboratório de Biologia Molecular de Bactérias (LBMB), IMPPG/CCS/UFRJ, onde $50 \mu \mathrm{L}$ do caldo contendo as células diluídos em 2,0mL de TSB foram reativadas conforme descrito acima. Todas as amostras foram, então, novamente avaliadas quanto à sua pureza a contaminantes. Em seguida, um inóculo com as cepas de $S$. aureus isoladas das amostras foi nária, as cepas estudadas e isoladas de leite proveniente de vacas portadoras de mastite subclínica na região apresentaram-se sensiveis a meticilina através do teste de triagem em placa utilizando $25 \mu \mathrm{g} / \mathrm{mL}$ dessa droga. Embora a concentração de meticilina utilizada no presente trabalho para testar as cepas isoladas de mastite bovina tenha sido diferente das concentrações utilizadas sobre cepas, também de origem bovina, por outros autores (Devriese, 1975 ; Matsunaga et al., 1990), esta concentração $(25 \mu \mathrm{g} / \mathrm{mL})$ corresponde aos testes padronizados para cepas de origem humana e utilizadas na detecção do clone brasileiro de $S$. aureus meticilina-resistente (MRSA) III::B:A (Teixeira et al., 1996; Teixeira et al. 1995). As amostras de Matsunaga et al. (1990) foram sensíveis à meticilina na concentração de $6,25 \mu \mathrm{g} / \mathrm{mL}$ e as amostras de Devriese (1975) foram sensíveis a diferentes concentrações da droga, ou seja, 20, 50 e $100 \mu \mathrm{g} / \mathrm{mL}$.

No Quadro 1 , um total de 128 cepas de $S$. aureus foram testadas frente aos antimicrobianos e foi demonstrado que a maioria das drogas apresentaram efeito inibidor satisfatório, exceto para PEN, OXA, AMP e AMO. As drogas com maior percentual de cepas com grau de resposta intermediária foram ERI e CFO, com $27,7 \%$ e $22,8 \%$ respectivamente. As drogas que apresentaram $100 \%$ de inibição sobre as cepas foram GEN, SUL e VAN. Resultados semelhantes de sensibilidade foram obtidos com AMI, CFL e CLO (96\%, 96\% e 93\%

Quadro 1: Resultados de antibiogramas de 128 cepas de Staphylococcus aureus provenientes de leite mastítico, da região Norte /Noroeste do Estado do Rio de Janeiro, durante os anos de 1996 e 1997

\begin{tabular}{|c|c|c|c|c|c|c|c|c|c|c|c|c|c|c|c|}
\hline $\begin{array}{l}\text { Droga } \\
\text { Perfil }\end{array}$ & PEN & OXA & AMP & GEN & AMI & TET & CFL & ERI & $\mathrm{CLI}$ & LIN & SUL & AMO & CLO & VAN & CFO \\
\hline $\begin{array}{c}\% \mathrm{R} \\
\% 1 \\
\% \mathrm{~S} \\
\end{array}$ & $\begin{array}{c}96,1 \\
- \\
3,9 \\
\end{array}$ & $\begin{array}{c}87,0 \\
5,3 \\
7,6 \\
\end{array}$ & $\begin{array}{c}99,2 \\
- \\
0,8 \\
\end{array}$ & $\begin{array}{c}- \\
100 \\
\end{array}$ & $\begin{array}{r}3,4 \\
- \\
96,6 \\
\end{array}$ & $\begin{array}{r}9,5 \\
5,5 \\
85,0 \\
\end{array}$ & $\begin{array}{c}0,9 \\
3,1 \\
96,0 \\
\end{array}$ & $\begin{array}{r}4,0 \\
27,5 \\
68,5 \\
\end{array}$ & $\begin{array}{r}4,0 \\
9,0 \\
87,0 \\
\end{array}$ & $\begin{array}{r}2,7 \\
8,1 \\
89,2 \\
\end{array}$ & $\therefore$ & $\begin{array}{c}88,2 \\
9,8 \\
2,0\end{array}$ & $\begin{array}{c}3,1 \\
3,1 \\
93,8 \\
\end{array}$ & $\begin{array}{c}- \\
100\end{array}$ & $\begin{array}{r}5,5 \\
22,8 \\
71,6\end{array}$ \\
\hline $\begin{array}{l}X A-C \\
M P-A \\
\text { EN - }\end{array}$ & $\begin{array}{l}\text { na } \\
\text { aa } \\
\text { ina } \\
\text { icina }\end{array}$ & & $\begin{array}{l}R-R \\
\text { CFL } \\
\text { ERI - }\end{array}$ & $\begin{array}{l}\text { efalot } \\
\text { itromi }\end{array}$ & & $\begin{array}{l}\mathrm{SU} \\
\mathrm{CL} \\
1-\end{array}$ & med & $\begin{array}{l}\text { cina } \\
\text { rim } \\
\text { enicol } \\
\text { a }\end{array}$ & & $\begin{array}{l}F O-C \\
\text { IN - Lin }\end{array}$ & micina & & & $\mathrm{II}-\mathrm{Al}$ & $\begin{array}{l}\text { xicilina } \\
\text { acina }\end{array}$ \\
\hline
\end{tabular}

preparado em $50 \mu \mathrm{L}$ do caldo e inoculado em placas de ágar Mueller Hinton contendo $25 \mu \mathrm{g}$ de Meticilina (Sigma, cat. $\mathrm{n}^{\circ}$ M6535) por placa, e distribuído uniformemente com bastão de vidro tipo "hockey" sobre a superfície do ágar. Após lacrar as placas com filme de PVC, foi feita a incubação a $37^{\circ} \mathrm{C}$ por 24 horas em estufa.

\section{Preparação da Meticilina para o Teste Met 25}

A concentração inicial de meticilina foi de $1 \mu \mathrm{g} / \mathrm{mL}$ (solução I). Foram utilizados $0,012 \mathrm{~g}$ de meticilina em $1,0 \mathrm{~mL}$ de água bidestilada autoclavada (solução II). Para prepararmos 500 $m L$ de TSA (Tryptic Soy Agar) (Difco, cat. $n \div 0369-01-4$ ) contendo meticilina foram utilizados $1,25 \mathrm{~mL}$ da solução II.

\section{Resultados e discussão}

\section{Leitura do resultado do teste Met-25}

As primeiras cepas de Staphylococcus aureus meticilina-resistentes, isoladas de leite de vacas mastíticas, datam de 1972 (Devriese et al., 1972). Apesar da penicilina e dos seus derivados semi-sintéticos serem utilizados na prática veteri- respectivamente) seguidos de LIN (89,2\%) e CLI (87\%), TET (85\%), CFO (71,6\%) e ERI $(68,5 \%)$.

Provavelmente em conseqüência do uso indiscriminado de drogas b-lactâmicas nas regiões estudadas, estes resultados indicam um índice de resistência às referidas drogas considerado alto, embora um pequeno percentual tenha mostrado sensibilidade (OXA 7,6\% S; AMP 0,8\% S), com exceção para os b-lactâmicos CFL e CFO (0,9\% R, 3,1\% । e $96,0 \%$ S para a primeira droga e $5,5 \% R, 22,8 \%$ । e $71,6 \% \mathrm{~S}$ para segunda droga) que ainda não fazem parte do uso cotidiano pelos pecuaristas ao combate à mastite bovina em grau tão elevado quanto às demais drogas do grupo dos b-lactâmicos, embora já estejam disponíveis no mercado da região (Folly et al., 1998). Bezec (1998) constatou diferentes padrões de sensibilidade a antimicrobianos utilizados nos tratamentos de rebanhos leiteiros e mostrou que a droga mais eficaz, in vitro, para bactérias do gênero Staphylococcus foi a cefalotina, o que vem de encontro com o resultado encontrado neste trabalho para a referida droga. Entretanto, observamos a presença de cepas na faixa intermediária de sensibilidade às ultimas drogas do grupo dos b-lactâmicos citadas (principalmente cefoxitina), o que pode denotar uma preocupação quan- 
to a futuras cepas resistentes também a estes medicamentos. Os estudos de Matsunaga et al. (1990) com amostras de $S$. aureus de vacas mastíticas também mostraram a ação de antimicrobianos sobre este microrganismo e concluíram que as drogas mais afetadas por b-lactamase produzidas por cepas bacterianas foram penicilina $G$ e ampicilina, o que, possivelmente, tenha influenciado nos resultados obtidos no presente trabalho, com as referidas drogas. O uso indiscriminado dessas drogas na região pode acarretar o aparecimento de cepas resistentes da bactéria oferecendo risco à população, principalmente crianças e idosos na área rural, que fazem uso de leite in natura e industrializado em sua dieta, por estarem consu-

\section{Referências bibliográficas}

BAUER, A.W., KIRBY, W.M.M., SHERRIS, J.C., TURCK, M. Antibiotic susceptibility testing by a standardized single disck method. The American Journal of Clinical Pathology, v. 45, n. 4, p. 493-496, 1966.

BELKUM, A. Van, ERICKSEN, N.H.R., SIJMONS, M., LEEUWEN, W.Van, BERGH, M. Van Den, KLUYTMANS, J, ESPERSEN, F., VERBURGH, $\mathrm{H}$. Coagulase and protein A polymorphisms do not contribute to persistence of nasal colonisation by Staphylococcus aureus. Journal of Medical Microbiology, v. 46, p. 222-232, 1997.

BIOMERIÉUX. ApiStaph Système d'identification des staphylocoques et microcoques. Lyon, França. p. 1-8, 1993.

BEZEC, D.M. Genus identification and antibiotic susceptibility patterns of bacterial isolates from cows with acute mastitis in a practice population. Journal of the American Veterinary Medicine Association, v. 212, n. 3, p. 404-406, 1998.

CMT-FATEC. California Mastitis Test : reagente químico. FATEC S.A. Licenciado Min. da Agricultura sob no0834, São Paulo-SP.

DEGRAVES, J.F., FETROW, J. Economics of Mastitis and Mastitis Control. In: Update on bovine mastitis. The Veterinary Clinics of North America: Food Animal Practice, v. 9, p. 421-434, 1993.

DE LENCASTRE, H. FIGUEIREDO, A.M.S., URBAN, C. RAHAL, J. TOMASZ, A. Multiple mechanisms of methicilin resistence and improved methods for detection in clinical isolates of Staphylococcus aureus. Antimicrobial Agents and Chemotherapy, v. 35, p. 632-639, 1991.

DEVRIESE. L.A, VANDAMME, L.R., FAMEREE, L. Methicillin (cloxacillin)resistance Staphylococcus aureus strains isolated from bovine mastitis cases. Zentralblat Veterinarmedizin [B], v. 19, n. 7, p. 598-605, 1972.

DEVRIESE. L.A. Epidemiology of methicillin-resistant Staphylococcus aureus in dairy herds. Research in Veterinary Science, v. 19, p. 23-27, 1975.

DIFCO MANUAL. Dehydrated Culture Media and Reagents for Microbiology, 10. ed., Difco Laboratories Inc., Detroit, Michigan, EUA. 1155 p., 1994.

FILIPPSEN, L.F., MOREIRA, F.B., SAKASHITA, A.T., ZAFALON, L.F., BRITO, J.R.F. Prevalência da Mastite subclínica em rebanhos leiteiros no Norte do Paraná. Anais do XXIV Congresso Brasileiro de Medicina Veterinária, Goiânia, GO, 1996.

FOLLY, M.M., MOTTA, O.V., MACHADO, S.C.A. Controle da mamite bovina na região Norte e Noroeste do Estado do Rio de Janeiro. Boletim Técnico, v. 3, n. 2, p. 1-17. Universidade Estadual do Norte Fluminense, FENORTE, CCTA. Secretaria de Estado de Ciência e Tecnologia, Governo do Estado do Rio de Janeiro, 1998.

GERMANO, P.M.L., GERMANO, M.I.S. Higiene do leite: aspectos gerais das mastites. Higiene Alimentar, v. 9, n. 36, p. 12-16, 1995. mindo produto com possível presença de resíduo dessas drogas (Machado, 1998), além do risco de se contaminarem com estas cepas bacterianas.

\section{Conclusão}

As cepas de Staphylococcus aureus isoladas de leite mastítico na região Norte/Noroeste do Estado do Rio de Janeiro não mostraram resistência a meticilina através do teste de triagem em placa utilizando $25 \mu \mathrm{g} / \mathrm{mL}$ dessa droga in vitro. As cepas apresentaram um elevado grau de resistência à penicilina e aos seu derivados b-lactâmicos semi-sintéticos, com exceção a cefalotina e cefoxitina.

HACKBARTH, C.J., CHAMBERS, H.F. Methicillin-resistant staphylococci: genetics and mechanisms of resistance. Antimicrobial Agents and Chemotherapy, v. 33, n. 7, p. 991-994, 1989.

HUMMEL, R., LEHMANN, G. Bovine Coagulase-Negative Staphylococci: Biochemistry and Polymerase Chain Reaction. Acta Vet. Brno, v. 63, p. 133-139, 1994.

LABORCLIN. Produtos para Laboratórios. Pinhais, PR.

LANGLOIS, B.E., PARLINDUNGAN, A.K., HARMON, R.J., AKERS, K. Biochemical Characteristics of Staphylococcusspecies of human and bovine origin. Journal of Food Protection, v. 53 n. 2, p. 119-126, 1990.

MACHADO, S. A.C. Utilização de métodos rápidos de análise para determinação da incidência da contaminação por resíduos de antibióticos no leite pasteurizado comercializado na Região Norte/Noroeste do Estado do Rio de Janeiro. 1998. 67 p. Tese (Mestrado) - Universidade Estadual do Norte Fluminense.

MATSUNAGA, T., YOSHIDA, T., KAMATA, S., UCHIDA, K. Identification of staphylococci from bovine mastitis and an examination of their susceptibility to antibiotics and beta-lactamase production. Nippon Juigaku Zasshi, v. 52, n. 6, p. 1219-1227, 1990.

MICROBIOLOGY MANUAL MERCK. Darmstadt, Holanda. 405 p. Edição 1996/97.

NEU, H.C. The crisis in antibiotic resistance. Science, v. 257, p.10641072, 1992.

OWENS, W.E., WATTS, J.L. Effects of milk activity of antimicrobiotics against Staphylococcus aureus isolated from bovine udders. Journal of Dairy Science, v. 70, n. 9, p. 1946-1951, 1987.

OWENS, W.E., RAY, C.H., WATTS, J.L., YANCEY, R.J. Comparison of success of antibiotic therapy during lactation and results of antimicrobial susceptibility tests for bovine mastitis. Journal of Dairy Science, v. 80, n. 2, p. 313-317, 1997.

RUBIN, R.J., HARRINGTON, C.A., POON, A., DIETRICH, K., GREENE, J.A., MOIDUDDIN, A. The economic impact of Staphylococcus aureus infection in New York City hospitals. Emerging Infectious Diseases, v. 5, n. 1, p. 9-17, 1999.

SARAN, A. Mastitis bovina: enfermedades de la ubre y su control en Israel. Inst. Veterinario Kimron, Estado de Israel, p. 72, 1986. Traduzido.

TEIXEIRA, L.A., RESENDE, C.A., ORMONDI, L.R., ROSENBAUM, R., FIGUEIREDO, A.M.S., De LENCASTRE, H., TOMASZ, A. Geographic spread of epidemic multiresistant Staphylococcus aureus clone in Brazil. Journal of Clinical Microbiology, v. 33, n. 9, p. 2400-2404, 1995.

TEIXEIRA, L.A., LOURENÇO, M.C, FIGUEIREDO, A.M.S. Emergence of a methicilin-resistant Staphylococcus aureus clone related to the Brazilian epidemic clone III::B:A causing invasive disease among AIDS patients in a brazilian hospital. Microbial Drug Resisance, v. 2, n. 4, p. 393-399, 1996.

TYLER, J.W. Treatment of Gram-negative mastitis. $28^{\text {th }}$ Annual Convention Proceedings, Am. Assoc. of bovine Practitioners. The bovine proceedings, n. 28, p. 94-96, 1996. 